Harvey Rosen, Department of Mathematics, University of Alabama, Tuscaloosa, AL 35487. e-mail: hrosen@gp.as.ua.edu

\title{
POROSITY IN SPACES OF DARBOUX-LIKE FUNCTIONS
}

\begin{abstract}
It is known that the six Darboux-like function spaces of continuous, extendable, almost continuous, connectivity, Darboux, and peripherally continuous functions $f: \mathbb{R} \rightarrow \mathbb{R}$, with the metric of uniform convergence, form a strictly increasing chain of subspaces. We denote these spaces by C, Ext, AC, Conn, D, and PC, respectively. We show that C and D are porous and $\mathrm{AC}$ and Conn are not porous in their successive spaces of this chain.
\end{abstract}

The porosity of special sets in spaces of Darboux-like functions has been studied, for example, in [10] and [11]. For functions $f: \mathbb{R} \rightarrow \mathbb{R}$, it is known that $\mathrm{C} \subset$ Ext $\subset \mathrm{AC} \subset$ Conn $\subset \mathrm{D} \subset \mathrm{PC}$ [12]. Each function space we study will have on it the metric $d$ of uniform convergence defined by $d(f, g)=$ $\min \{1, \sup \{|f(x)-g(x)|: x \in \mathbb{R}\}\}$. In [2, thm 7, p.445], Bruckner and Ceder show that if $f \in \operatorname{cl}(\mathrm{D})$ and the graph of $f$ is dense in $\mathbb{R}^{2}$, then $f \in \operatorname{cl}(\mathrm{Conn})$ and Conn is dense in each open ball in cl(D) with radius $\leq 1$ and centered at $f$. Therefore Conn is somewhere dense in $\mathrm{D}$, and it follows that Conn is not porous at some point of $\mathrm{D}$. However, we show Conn is a boundary set in $\mathrm{D}$. We also show $\mathrm{C}$ is porous in Ext, $\mathrm{D}$ is porous in $\mathrm{PC}$, but $\mathrm{AC}$ is not porous in Conn. Whether or not Extis porous in AC is left as an open problem.

A subset $K$ of $\mathbb{R}^{2}$ is said to be bilaterally dense (resp. bilaterally c-dense) in itself if for each $z \in K$, each open square which has a vertical side bisected by $z$ contains infinitely many (resp. c-many) points of $K$.

For a function $f: \mathbb{R} \rightarrow \mathbb{R}$ we define:

1. $f \in \mathrm{PC}$ if the graph of $f$ is bilaterally dense in itself.

2. $f \in \mathrm{D}$ if $f(J)$ is connected for each connected set $J \subset \mathbb{R}$.

Key Words: porosity, spaces of continuous, extendable, almost continuous, connectivity, Darboux, peripherally continuous functions

Mathematical Reviews subject classification: 26A15, 54C08, 54C35

Received by the editors July 31, 1999 
3. $f \in$ Conn if the graph of the restriction $f \uparrow J$ is connected for each connected set $J \subset \mathbb{R}$.

4. $f \in \mathrm{AC}$ if each open neighborhood in $\mathbb{R}^{2}$ of the graph of $f$ contains the graph of a continuous function $g: \mathbb{R} \rightarrow \mathbb{R}$.

5. $f \in$ Ext if there is a function $F: \mathbb{R}^{2} \rightarrow \mathbb{R}$ such that $F(x, 0)=f(x)$ for all $x \in \mathbb{R}$ and the graph of $F\lceil J$ is connected for each connected set $J \subset \mathbb{R}^{2}$.

For $\epsilon>0, S_{\epsilon}(f)=\{(x, y): x \in \mathbb{R}$ and $|y-f(x)|<\epsilon\}$ denotes the $\epsilon$-strip about $f$. For a subset $K$ of $\mathbb{R}^{2}, \Pi_{1}(K)$ denotes the $x$-projection of $K$ and $K_{x}=K \cap \Pi_{1}^{-1}(x)=K \cap(\{x\} \times \mathbb{R})$. Suppose $A$ and $B$ are intervals in $\mathbb{R}$. A blocking set in $A \times B$ is a closed subset $K$ of $A \times B$ which meets every continuous function from $A$ into $B$ and which misses some function from $A$ into $B$. A function $f: A \rightarrow B$ is almost continuous relative to $A \times B$ if and only if it meets every blocking set in $A \times B$. Each blocking set in $A \times B$ contains a minimal blocking set $K$, and $\Pi_{1}(K)$ is a nondegenerate connected set and $K$ is a perfect set [8, thm 1, p. 182], [7, lem 3, p.126].

In a metric space $(X, d), B(x, r)$ denotes the open ball centered at $x$ with radius $r>0$. Let $M \subset X, x \in X$, and $r>0$. Then $\gamma(x, r, M)$ denotes the supremum of the set of all $s>0$ for which there exists $z \in X$ such that $B(z, s) \subset B(x, r) \backslash M . M$ is porous at $x$ if $\limsup _{r \rightarrow 0^{+}} \frac{\gamma(x, r, M)}{r}>0 . M$ is porous in $X$ if $M$ is porous at each $x \in X$. A porous set $M$ turns out to be a boundary set in $X$.

Let $\mathcal{A} \subset \mathcal{B}$ be consecutive spaces in the above chain of Darboux-like spaces. $\mathcal{B} \backslash \operatorname{cl}(\mathcal{A}) \neq \emptyset$ because of $\left[4\right.$, thm 9.10, p. 517] and because $y= \begin{cases}\sin \frac{1}{x} & \text { if } x \neq 0 \\ 0 & \text { if } x=0\end{cases}$ belongs to Ext $\backslash \operatorname{cl}(\mathrm{C})=$ Ext $\backslash \mathrm{C}$ and the characteristic function $\chi_{\mathbb{Q}}$ of the set $\mathbb{Q}$ of rational numbers belongs to $\mathrm{PC} \backslash \operatorname{cl}(\mathrm{D}) . \mathcal{A}$ is porous at each member of the open set $\mathcal{B} \backslash \operatorname{cl}(\mathcal{A})$. So to verify whether $\mathcal{A}$ is porous in $\mathcal{B}$, it suffices to check porosity at just the functions $f$ in $\mathcal{B}$ that are uniform limits of sequences $\left\langle f_{n}\right\rangle$ in $\mathcal{A}$.

Theorem 1. $C$ is porous in Ext.

Proof. According to the last observation, it suffices to show $\mathrm{C}$ is porous at $f \in$ Ext when $f$ is a uniform limit of a sequence in $C$. But then $f \in \mathrm{C}$. Let $0<r \leq 1$ and $x_{0} \in \mathbb{R}$. There exists $\delta>0$ such that $f\left(\left[x_{0}-\delta, x_{0}+\delta\right]\right) \subset$ $\left(f\left(x_{0}\right)-\frac{r}{8}, f\left(x_{0}\right)+\frac{r}{8}\right)$. As in the proof of Theorem 2 in [11], define $g: \mathbb{R} \rightarrow \mathbb{R}$ by 


$$
g(x)= \begin{cases}f(x) & \text { if } x \notin\left(x_{0}-\delta, x_{0}+\delta\right) \\ l_{1}(x) & \text { if } x \in\left[x_{0}-\delta, x_{0}\right] \\ \frac{r}{8} \sin \frac{1}{x-x_{0}}+f\left(x_{0}\right) & \text { if } x \in\left(x_{0}, x_{0}+\frac{\delta}{2}\right) \\ l_{2}(x) & \text { if } x \in\left[x_{0}+\frac{\delta}{2}, x_{0}+\delta\right]\end{cases}
$$

where $l_{1}$ and $l_{2}$ are linear functions such that $l_{1}\left(x_{0}-\delta\right)=f\left(x_{0}-\delta\right), l_{1}\left(x_{0}\right)=$ $f\left(x_{0}\right), l_{2}\left(x_{0}+\frac{\delta}{2}\right)=\frac{r}{8} \sin \frac{2}{\delta}+f\left(x_{0}\right)$, and $l_{2}\left(x_{0}+\delta\right)=f\left(x_{0}+\delta\right)$. Then $g \in$ Ext and $d(g, f)<\frac{r}{4}$. Therefore $B\left(g, \frac{r}{16}\right) \subset B(f, r)$ and $B\left(g, \frac{r}{16}\right) \cap \mathrm{C}=\emptyset$. Since $\gamma(f, r, C) \geq \frac{r}{16}, \limsup _{r \rightarrow 0^{+}} \frac{\gamma(f, r, C)}{r} \geq \frac{1}{16}>0$. This shows $\mathrm{C}$ is porous at $f$.

The next two results are analogous to Theorems 6 and 7 in $[2, \mathrm{p} .445]$. The proof of the second result depends on the part of Natkaniec's Theorem 1 in $[9$, p. 40] which states the following: Define a condition for any function $f:[0,1] \rightarrow \mathbb{R}$ this way: $(\alpha)$ for sufficiently small $\epsilon>0$ and for every blocking set $K$ in $[0,1] \times \mathbb{R}$, either $\operatorname{card}\left(\operatorname{dom}\left(K \cap S_{\epsilon}(f)\right)\right)=c$ or $(f(x)-\epsilon, f(x)+\epsilon) \subset K_{x}$ for some $x \in[0,1]$. Then $(\alpha) \rightarrow f \in \operatorname{cl}(\mathrm{AC})$.

He does not prove this part in [9], but he gave the following proof in a preprint of an earlier version of [9] without the Continuum Hypothesis.

Suppose the collection $\left\{K_{\alpha}: \alpha \in A\right\}$ of all blocking sets of $[0,1] \times \mathbb{R}$ is well ordered so that for each $\alpha \in A$, $\operatorname{card}\left(\left\{K_{\beta}: \beta<\alpha\right\}\right)<c$. For sufficiently small $\epsilon>0$, one can use transfinite induction to choose for each $\alpha \in A$ a point $\left(x_{\alpha}, y_{\alpha}\right) \in S_{\epsilon}(f) \cap K_{\alpha}$ such that if $\operatorname{card}\left(\operatorname{dom}\left(K_{\alpha} \cap S_{\epsilon}(f)\right)\right)=c$, then $x_{\alpha} \neq x_{\beta}$ for all $x \in[0,1]$. But if $\operatorname{card}\left(\operatorname{dom}\left(K_{\alpha} \cap S_{\epsilon}(f)\right)\right)<c$ and $(f(x)-\epsilon, f(x)+\epsilon) \subset\left(K_{\alpha}\right)_{x}$ for some $\beta<\alpha$, then choose $x_{\alpha}=x$ and either $y_{\alpha}=y_{\beta}$ whenever $x_{\beta}=x$ for some $\beta<\alpha$ or $y_{\alpha}=f(x)$ otherwise. The function $g:[0,1] \rightarrow \mathbb{R}$ defined by

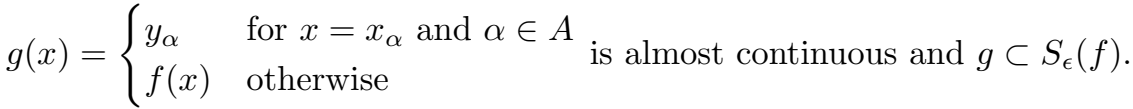

We can replace $[0,1]$ with $\mathbb{R}$ and we only have to check condition $(\alpha)$ holds for minimal blocking sets.

Theorem 2. If $g \in \operatorname{cl}($ Conn $)$ has a point $x_{0}$ of continuity, then there exist balls in $\mathrm{cl}(\mathrm{Conn})$ arbitrarily close to $g$ and containing no members of $A C$.

Proof. Let $0<\epsilon<1$. There is a $\delta>0$ such that whenever $\left|x-x_{0}\right|<\delta$, then $\left|g(x)-g\left(x_{0}\right)\right|<\frac{\epsilon}{4}$. In [5], Jastrzebski gives an example of a function $h$ from $[0,1]$ onto $[-1,1]$ such that $h \in \operatorname{Conn} \backslash \operatorname{cl}(A C)$. Let $f_{0}$ be a scaled-down copy of $h$ to $\left[x_{0}-\frac{\delta}{2}, x_{0}+\frac{\delta}{2}\right] \times\left[g\left(x_{0}\right)-\frac{\epsilon}{4}, g\left(x_{0}\right)+\frac{\epsilon}{4}\right]$ instead of $[0,1] \times[-1,1]$. Since $g \in \operatorname{cl}\left(\right.$ Conn), we can extend the domain of $f_{0}$ to all of $\mathbb{R}$ in such a way that $f_{0} \in$ Conn and $\left|f_{0}(x)-g(x)\right|<\epsilon$ for all $x$. Therefore there is a ball in cl(Conn) centered at $f_{0}$ and missing $\mathrm{AC}$ because $h \notin \mathrm{cl}(\mathrm{AC})$ implies $f_{0} \notin \mathrm{cl}(\mathrm{AC})$. 
Theorem 3. If $f \in \operatorname{cl}($ Conn $)$ is dense in $\mathbb{R}^{2}$, then $A C$ is dense in each open ball in $\mathrm{cl}($ Conn $)$ of radius $\leq 1$ with center $f$.

Proof. If $g \in \operatorname{cl}(\mathrm{Conn})$ is dense in $R^{2}$, then we initially show $g \in \operatorname{cl}(\mathrm{AC})$ according to [9] by verifying that $g$ obeys $(\alpha)$. Suppose $K$ is a minimal blocking set in $\mathbb{R}^{2}$. Let $S_{\epsilon}(K)$ denote the set obtained by replacing each point of $K$ by an open vertical interval of length $2 \epsilon$ centered at the point. Because $\Pi_{1}\left(S_{\epsilon}(K)\right)=$ $\Pi_{1}(K)$ is a nondegenerate interval [7], then by the Baire Category Theorem, $S_{\epsilon}(K)$ contains a rectangle $B$ with a vertical side of length $\epsilon$. Since $g \in$ $\operatorname{cl}($ Conn $)$ is dense in $\mathbb{R}^{2}$, then $\operatorname{card}(g \cap B)=c$ and so $\operatorname{card}\left(g \cap S_{\epsilon}(K)\right)=c$. That is, $\operatorname{card}\left(\operatorname{dom}\left(S_{\epsilon}(g) \cap K\right)=c\right.$ and therefore $g$ obeys $(\alpha)$.

Next, if $f_{0} \in \operatorname{cl}($ Conn $)$ and $d\left(f_{0}, f\right)<1$, then $f_{0}$ must be dense in $\mathbb{R}^{2}$ and so, as shown first, $f_{0} \in \mathrm{cl}(\mathrm{AC})$. This shows $\mathrm{AC}$ is dense in every open ball in $\operatorname{cl}(\mathrm{Conn})$ of radius $\leq 1$ and with center $f$.

The next result follows immediately from Theorem 3.

Theorem 4. AC is not porous in Conn.

Theorem 5. Conn is not porous in D [2], but Conn is a boundary set in D.

Proof. We must show that for each $f \in D$ and $r>0$, there exists $g \in$ $B(f, r) \backslash$ Conn. According to the proof of Theorem 6 in [2], for the case when $f \in \mathrm{D}$ and has a point of continuity, there exist balls in $\mathrm{D}$ arbitrarily close to $f$ and missing Conn. This implies $\mathrm{D} \backslash$ Conn has points arbitrarily close to $f$. (According to their proof, Conn is actually porous at this $f$.) Now consider the case when $f \in \mathrm{D}$ and has no point of continuity. Then the graph of $f$ is somewhere dense in $\mathbb{R}^{2}[6]$, [1]. Let $L$ be a closed line segment having positive slope and lying in a circular open neighborhood $U \subset \operatorname{cl}(f)$ with radius $\leq \frac{r}{2}$. A function $g: \mathbb{R} \rightarrow \mathbb{R}$ belonging to $\mathrm{D}$ can be obtained from $f$ by shifting vertically any points of $f \cap L$ off $L$ to points in $U$. Then $g \in B(f, r) \backslash$ Conn. Together both cases show Conn is a boundary set in D.

Theorem 6. $D$ is porous in $P C$.

Proof. Let $f \in \mathrm{PC}$. We may suppose $f \in \operatorname{cl}(\mathrm{D})$. Let $\mathcal{U}$ denote the class of all functions $f: R \rightarrow \mathbb{R}$ such that for every interval $J \subset \mathbb{R}$ and every set $A$ of cardinality less than $c, f(J \backslash A)$ is dense in $\left[\inf _{x \in J} f(x), \sup _{x \in J} f(x)\right]$. According to $[3$, thm 4.3 , p. 71$], \mathcal{U}=\operatorname{cl}(\mathrm{D})$. Then $f \in \mathcal{U} \subset$ PC. First suppose $f$ is not a constant function. For each sufficiently small $r>0$ with $r \leq 1$, there exists an interval $J=[a, b]$ such that $\frac{r}{4}<|f(a)-f(b)|<\frac{r}{2}$. For argument's sake, suppose $f(a)<f(b)$. Let $B=(a, b) \cap f^{-1}((f(a), f(b)))$. It follows that $f \uparrow B$ is bilaterally $c$-dense in itself. 
We show $B=E \cup F$, where $E$ and $F$ are disjoint bilaterally dense in itself sets and $f \uparrow E$ and $f \uparrow F$ are each dense in $f \uparrow B$. According to Theorem 3.2 in [3, pp. 67-68], since $f \in \mathcal{U}$, for each open interval $N, f^{-1}(N)$ is empty or $c$ dense in itself. Each such nonempty $f^{-1}(N)$, like $f^{-1}((f(a), f(b)))$, is actually bilaterally $c$-dense in itself. Let $P$ be a countable dense subset of the graph of $f \uparrow B$, and let $E=\Pi_{1}(P) . E$ is bilaterally dense in itself and $f \uparrow E=P$ is dense in $f \uparrow B$. Since $f((a, b) \backslash E)$ is dense in $\left[\inf _{x \in J} f(x), \sup _{x \in J} f(x)\right]$, the set $F=B \backslash E$ is bilaterally $c$-dense in itself and $f \uparrow F$ is dense in $f \uparrow B$. So $B=E \cup F$.

Define $g: \mathbb{R} \rightarrow \mathbb{R}$ by

$$
g(x)= \begin{cases}f(a) & \text { if } x \in E \\ f(b) & \text { if } x \in F \\ f(x) & \text { if } x \in \mathbb{R} \backslash B\end{cases}
$$

Then $g \in P C \backslash \mathcal{U}$, and $g \in B\left(f, \frac{r}{2}\right)$ because $|f(a)-f(b)|<\frac{r}{2} . \quad B\left(g, \frac{r}{8}\right) \subset$ $B(f, r) \backslash \mathrm{D}$ because $|f(a)-f(b)|>\frac{r}{4}$. Since $\gamma(f, r, D) \geq \frac{r}{8}$, it follows that $\limsup \sup _{r \rightarrow 0^{+}} \frac{\gamma(f, r, D)}{r} \geq \frac{1}{8}>0$ and so D is porous at $f$. When $f$ is a constant function with value $k$ and $0<r<1$, define

$$
g(x)= \begin{cases}k+\frac{r}{2} & \text { if } x \in \mathbb{Q} \\ k & \text { if } x \in \mathbb{R} \backslash \mathbb{Q} .\end{cases}
$$

Then $B\left(g, \frac{r}{4}\right) \subset B(f, r) \backslash D$, and it follows that $\mathrm{D}$ is porous at $f$.

\section{References}

[1] J. B. Brown, Nowhere dense Darboux graphs, Colloq. Math. 20 (1969), $243-247$.

[2] A. M. Bruckner and J. G. Ceder, On jumping functions by connected sets, Czech. Math. J. 22 (1972), 435-448.

[3] A. M. Bruckner, J. G. Ceder, and M. Weiss, Uniform limits of Darboux functions, Colloq. Math. , 15 (1966), 65-77.

[4] R. G. Gibson and T. Natkaniec, Darboux like functions, Real Anal. Exchange 22 (1996-97), 492-533.

[5] J. Jastrzebski, An answer to a question of R. G. Gibson and F. Roush, Real Anal. Exchange 15 (1989-90), 340-341. 
[6] F. B. Jones and E. S. Thomas, Jr., Connected $G_{\delta}$ graphs, Duke Math. J. 33 (1966), 341-345.

[7] K. R. Kellum, Sums and limits of almost continuous functions, Colloq. Math. 31 (1974), 125-128.

[8] K. R. Kellum and B. D. Garrett, Almost continuous real functions, Proc. Amer. Math. Soc. 33 (1972), 181-184.

[9] T. Natkaniec, On some problems concerning almost continuity, Real Anal. Exchange 17 (1991-92), 40-41.

[10] R. J. Pawlak, On some properties of the spaces of almost continuous functions, Internat. J. Math. Math. Sci. 19 (1996), 19-23.

[11] P. J. Pawlak, J. Kucner, and B. Swiatek, On small subsets of the space of Darboux functions, Real Anal. Exchange, 25 (1999-2000), 343-357.

[12] J. Stallings, Fixed point theorems for connectivity maps, Fund. Math. 47 (1959), 249-263. 